\title{
DISEÑO ORIENTADO A LA OPTIMIZACION EN ENVOLVENTES LAMINARES DE FLEXIÓN ACTIVA
}

Ma. Luciana Gronda, Mauro Chiarella, Martín Veizaga.

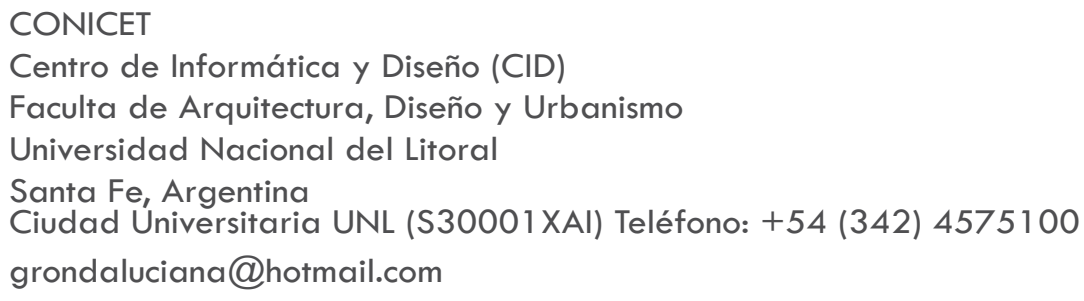

Palabras clave: Materialidad Digital; Optimización; form-finding; flexión activa; envolventes laminares.

\section{Resumen:}

En la Materialidad Digital, como estado actual del diseño arquitectónico, el enfoque de diseño orientado a la optimización de los recursos (materiales y energéticos) y la instrumentalización de la flexión activa introducen un nuevo nivel de integración en diseño arquitectónico. La necesaria especificación del comportamiento material revindica las históricas técnicas del form-finding como antecedente metodológico para la exploración material. La experimentación empírico-analítica, con modelos físicos para la concepción lógica de la forma compleja, se amplifica con la implementación del cálculo numérico computacional y sus posibilidades de simulación y evaluación digital.

El objetivo de la presentación es: analizar casos de estudio que verifiquen las ventajas estructurales que pueden ser generadas mediante la flexión elástica activa en envolventes laminares; sobre la base de las estrategias formales e investigación en diseño integral; y construir una mirada crítica sobre el avance de los procesos computacionales en la materialidad arquitectónica.

El supuesto subyacente es que, en los procesos integrales las decisiones en relación a la forma revisan simultáneamente atributos geométricos, estructurales y materiales. Dicho marco incluye procesos de modelado, análisis y fabricación. Se identifican los métodos que presentan potencial para repensar el diseño y avanzan en rigurosidad y precisión técnica a la vez que constituyen nuevos procedimientos y formas de abordaje para la materialidad arquitectónica.

\section{Introducción}

La arquitectura y la geometría siempre han estado intrínsecamente vinculadas. Sin embargo, su relación operacional se ha reforzado drásticamente con el reciente advenimiento del diseño computacional y las técnicas de fabricación digital. Estos desarrollos son dependientes recíprocamente, ya que la fabricación digital de componentes arquitectónicos complejos suscita la necesidad de avanzadas estrategias geométricas $y$, a cambio, los potenciales de la computación geométrica parten de la necesidad de eficiencia en la producción de formas complejas. Aunque actualmente confinado al dominio exclusivo de los especialistas, tales prácticas geométricas avanzadas comienzan a incluir un grupo profesional mucho más amplio e interdisciplinario, incluyendo arquitectos, ingenieros, informáticos y matemáticos. Su autoría en el desarrollo creativo de herramientas computacionales específicas a la arquitectura puede revolucionar el proceso de diseño, desde el descubrimiento conceptual inicial hasta su fabricación y construcción final.

En este marco, el progresivo perfeccionamiento del software y el diseño paramétrico, de lógica matemático-algorítmica, incrementan la complejidad en el manejo de la información iterativa y asociada a partir de algoritmos de alta precisión para la optimización. Por otro lado, las herramientas de fabricación digital amplían su alcance para experimentar, en la última década, un giro decisivo hacia lo material que potencia de manera inédita las capacidades productivas disciplinares. Este Giro paradigmático de lo digital en arquitectura hacia una nueva condición material, re- 
fiere de manera directa a los cambios en la manera en que comprendemos, producimos y usamos lo material en arquitectura. (PICON, Antoin; 2006; SENNETT, Richard, 2009).

\section{Marcos Conceptuales Emergentes}

\subsection{Materialidad Digital}

El concepto propuesto por Gramazio \& Kohler (2008) refiere al complejo entretejido posible entre la programación computacional y la fabricación digital; los datos y la materia. El término es utilizado para describir una transformación emergente en las lógicas materiales en arquitectura, refiere fundamentalmente a los cambios en la condición material, sus modos de concebirla y de operar en ella. Como estado actual del debate pero también como postura crítica frente al proceso disciplinar de lo que, a lo largo de las últimas décadas, ha implicado lo digital en arquitectura. Parte de una provocación reflexiva hacia cierta inercia de la tradicional cultura arquitectónica, estableciendo novedosos vínculos que amplían y enriquecen la relación entre la ideación, la tecnología, el entorno construido y el medio ambiente. Materialidad Digital no pretende iniciar un nuevo principio sobre el cual formalizar una nueva definición disciplinar, sino constituir un marco amplio de referencia con fuentes que den cuenta de los desplazamientos y las continuidades en los modos de comprender el cambio al que viene asistiendo la disciplina arquitectónica en las últimas décadas. Brinda las condiciones de comprensión y un marco de trabajo posible para encontrar herramientas y metodologías prácticas desde donde operar, con una visión enfocada a mejorar la relación entre el entorno construido y el medio ambiente (en el sentido más amplio).

\subsection{Performance o Diseño orientado a la optimización}

Algunos de los primeros autores que comienzan a abordar el concepto de Performance desde la arquitectura como un nuevo paradigma de diseño son: Kolarevic y Malkawi (2005); Hensel y Menges (2008).

Branco Kolarevic, señala que en arquitectura la performance se desarrolla en formas indeterminadas, en contraste con la rigidez predeterminada. Advierte el hecho de que el concepto de rendimiento va mucho más allá de las dimensiones estéticas y funcionales.

Achim Menges, aplica la teoría de los sistemas de auto-organización al diseño estructural, el form-finding y la investigación sobre los materiales. El término Performance es utilizado por Menges como una conflación entre "forma" y "rendimiento", es la cualidad que poseen los sistemas materiales para actuar de manera activa por deformación. Lo que Lienhard (2014) denomina, en su tesis doctoral, como Bending active Structures (estructuras de plegado o flexión activa) para el uso de la deformación elástica como potencialidad en los sistemas estructurales estáticos o cinéticos, logradas a partir del enfoque integral.

El enfoque del diseño orientado a la optimización radica en la forma en que el rendimiento material se entiende e instrumentaliza para revertir el paradigma moderno de la revolución industrial, del material homogéneo, estático, inerte, estandarizado, y de composición uniforme, isotrópico, con propiedades idénticas o muy similares en todas sus direcciones, que ha condicionado al material en su uso pasivo, con tipologías y disposiciones espaciales y estructurales predefinidas, ancladas a los paradigmas de la mecánica clásica.

Términos como "performance-oriented design" (diseño orientado al rendimiento), o "performance-oriented architecture" (arquitectura orientada al rendimiento) son propuestos como un enfoque integrador entre el diseño arquitectónico, el entorno construido y el medio ambiente (HENSEL, Michael Ulrich. 2013). Se destacan, además, las producciones de Sergio Araya (2011); Neri, Oxman (2010); Rivka, Oxman (2008), entre otros.

Neri Oxman avanza en la conceptualización misma de la forma y sugiere que en el diseño de 
la forma optimizada (performance) operan tres grandes dimensiones: el material, la estructura y la geometría, las cuales informan el diseño. Oxman sostiene que la separación institucionalizada entre forma, estructura y material, profundamente arraigada en la división metodológica entre modelado, análisis y fabricación, históricamente ha priorizado la forma por sobre el material y la estructura, dando lugar a toda una generación de tendencias formalistas en arquitectura. Consideraciones desde el dominio de la geometría, la materialidad, la fabricación, la lógica de montaje, el comportamiento y el medio ambiente (con algunas superposiciones) se yuxtaponen para dirigir el proceso de form-finding.

Cada autor construye su propia línea de investigación, pero todos comparten que una de las preocupaciones actuales de la arquitectura, como práctica material, es la forma en que los entornos construidos y naturales interactúan entre sí.

\subsection{Form-finding + Cálculo y simulación computacional}

Las actuales técnicas de simulación digital recuperan la lógica de las estrategias del form-finding. Esta herramienta metodológica empírico-física para la concepción lógica de la forma compleja, con claros antecedentes en la historia disciplinar, permiten a los arquitectos retomar ciertas lógicas de trabajo contenidas en su rol original, emprendiendo tanto el diseño como la producción. Ejemplos con modelos paramétricos físicos en el S.XX, emprendidos igualmente por arquitectos e ingenieros, son las primeras investigaciones estructurales de Vladímir Grigórievich Shújov (Ingeniero, 1853-1939); los modelos tridimensionales de Antonio Gaudí (Arquitecto, 1852-1926) y su original instrumento de proyección arquitectónica: la maqueta estereostática o maquetas polifuniculares colgadas para la Capilla Colonia Güell; Las estructuras a compresión de Félix Candela (Arquitecto, 1910-1997) y Eduardo Torroja Miret (Ingeniero, 1899-1961); Las pompas de jabón para el estudio de las estructuras neumáticas de Frei Otto (Arquitecto, profesor y teórico, 19252015), los modelos de cáscara de Heinz Isler (Ingeniero, 1926-2009) y las cúpulas geodésicas de Buckminster Fuller (Diseñador, arquitecto e inventor, 1895-1983). Constituyen el precedente del diseño paramétrico en su versión analógica de la actual técnica del form finding digital.

La complejidad de los sistemas estructurales y los procedimientos form-finding requieren un análisis numérico preciso. El método se basa en la Simulación de elementos finitos (MEF en castellano o FEM en inglés). Las rutinas de cálculo de elementos finitos (no lineales) han avanzado tanto últimamente que cada vez es más común integrarlos en el proceso de diseño a través de muchos softwares como SOFISTIK, entre otros.

\subsection{Otto, el ICD + ITKE = ITECH y sus pabellones}

Frei Otto funda en Berlín el Instituto de Estructuras Ligeras (Institut für leichte Flächentragwerke, IL), impartiendo además una vocación multidisciplinar en el contexto de su interés por los sistemas naturales, los modelos físicos experimentales, la geometría, la matemática iterativa y la irregularidad. El trabajo pionero que emprende Frei Otto en el IL es continuado desde la Universidad a través del nuevo programa: Integrative Technologies and Architectural Design Research (ITECH) llevado conjuntamente por Menges en el Instituto de Diseño Computacional (ICD) y Jan Knippers del Instituto de Estructuras Construcción y Diseño Estructural (ITKE). Ambos institutos trabajan de manera conjunta persiguiendo un enfoque multidisciplinario de investigación basada en una intensa colaboración con ingenieros estructurales, informáticos, científicos y biólogos materiales.

\subsection{Flexión Activa / Active Bending}

Según Lienhard (2014) la flexión activa es la instrumentalización de la deformación elástica para conformar estructuras a partir de elementos lineales o planos y aportarles rigidez mediante la pretensión. Este tipo de sistema estructural utiliza deformaciones a gran escala como estrategia 
formadora y autoestabilizadora (Knippers et al., 2011, Lienhard et al., 2013, 2014, Schleicher et al., 2015). Las estructuras de flexión activa se pueden dividir en dos categorías en relación a las dimensiones geométricas de sus elementos constitutivos: la línea (sistemas 1D) y el plano sistemas 2D). La elección por el plano responde a que existe un extenso conocimiento y experiencia para sistemas 1D y las estructuras laminares aún no han recibido mucha atención y se consideran difíciles de diseñar. Las placas se deforman principalmente a lo largo del eje de inercia más débil y, por lo tanto, no pueden adaptarse fácilmente a geometrías complejas. Sin embargo, esta limitación utilizada estratégicamente, ofrece no sólo más control sobre el proceso de formación global, sino que también puede usarse para informar a las partes individuales de la estructura ensamblada en base a las características de la forma general. Esto significa esencialmente que es posible comenzar primero con una forma global deseada y luego resolver el equilibrio entre la forma y la fuerza de sus partes, garantizando que las tensiones permanezcan dentro del rango de trabajo permitido del material.
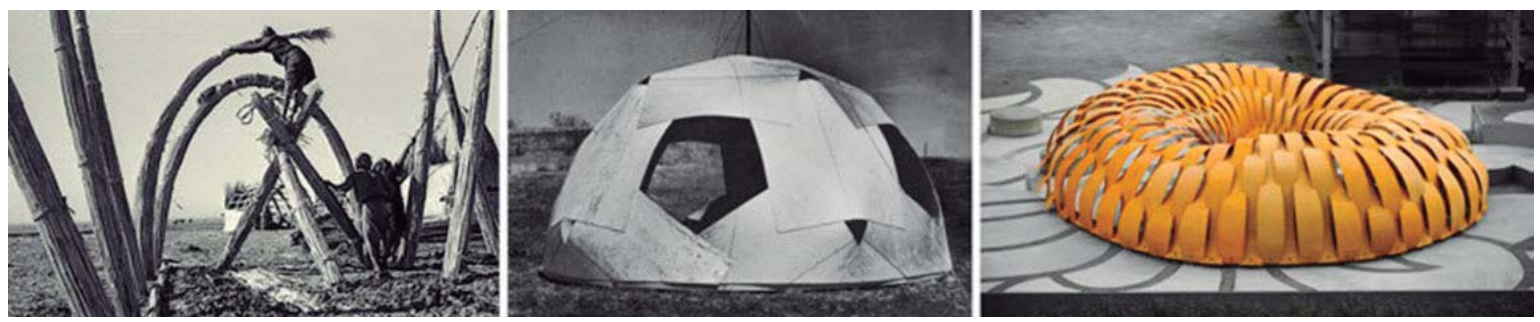

Figura 1.a: Mudhif. Casa de caña en los pantanos del sur de Irak en proceso traditional de construction artesanal. Figura 1.b: Plydome Buckminster Fuller, 1959. Figura 1.c: ICD/ITKE Research Pavilion 2010. Fuente: Lienhard Julian, Phd Thesis, 2014.

Las estructuras de flexión activa persiguen tres tipos de enfoques, un enfoque basado en el comportamiento, uno basado en la geometría y un tercer enfoque integral (Lienhard et al., 2013). Mientras que la primera imagen se refiere al uso tradicional e intuitivo de la flexión durante el proceso de construcción y se basa sólo en la experiencia práctica con respecto al comportamiento de deformación del material de construcción usado (Fig. 1. a), las dos últimas categorías describen una toma más científica del Diseño de estructuras de flexión activa. En este caso, las técnicas experimentales y analíticas de determinación de forma se realizaron de antemano y luego informaron al proceso de diseño.

Un ejemplo de las estructuras de flexión activa con materiales laminares, construido siguiendo un enfoque de diseño basado en la geometría, es el plydome de Buckminster Fuller. Este principio de construcción se basa en aproximar la geometría básica de una esfera a un poliedro regular. Sus bordes y ángulos se utilizan para superponer múltiples placas en un patrón de mosaico espacial, doblando y fijando las placas en sus esquinas (fig. 1.b). La estructura resultante está hecha de placas idénticas unidas entre sí colocando tornillos en posiciones predefinidas. Aunque esta técnica le permitió a Fuller construir una forma esférica de doble curvatura a partir de un material inicialmente plano y estandarizado, a partir de curvas simples, esta metodología también presentaba varias deficiencias. Se limita a formas poliédricas básicas, sólo por los ángulos repetitivos era posible utilizar placas idénticas. En su momento, Fuller se vio obligado a calcular la necesaria superposición de las placas y la posición exacta de los agujeros pre-perforados matemáticamente, la única manera de calibrar estos datos y mejorar los detalles fue produciendo plydomes en serie.

Un ejemplo destacado que asume un enfoque de diseño integral en estructuras laminares de flexión activa es el Pabellón de Investigación ICD / ITKE 2010 (Fig. 1.c). Este proyecto comenzó con pruebas de laboratorio intensivas para comprender el comportamiento del material y los limites estructurales del contrachapado. Los resultados de estos experimentos físicos se integraron como restricciones en herramientas de diseño paramétrico y se utilizaron para calibrar simulaciones de 
elementos finitos. La sincronización de los estudios físicos y digitales garantizó que las técnicas de búsqueda de formas, form-finding techniques, proporcionaran una descripción precisa del comportamiento del material $y$, al mismo tiempo, retroalimenten la geometría digital con los datos del análisis de la estructura. Este proyecto fue un medio para recrear el proceso de curvado material, simulando la deformación de cada tira en un sistema interconectado y elásticamente pretensado (Lienhard et al., 2012).

\section{Metodología}

Se define y describe el enfoque de diseño para estructuras de flexión activa en envolventes laminares, a partir del análisis de tres estudios de caso. Se revisa el enfoque de diseño en las estructuras a flexión activa y los desarrollos materiales de tales estructuras. Se diferencian tres enfoques: el enfoque basado en el comportamiento (EMPIRICO), el enfoque basado en la geometría (NUMERICO) y la investigación actual (INTEGRAL) que busca integrar los dos a partir del cálculo numérico computacional. Al categorizarlos, este trabajo pretende contribuir a un desarrollo emergente. La instrumentalización de la flexión activa en estructuras laminares introduce un nuevo nivel de integración en el diseño arquitectónico, a través de la especificación precisa del comportamiento material y de las estrategias computacionales para operar desde un enfoque de diseño orientado a la optimización.

\section{Estudio de casos}

El límite principal para el potencial formal de las estructuras de flexión activa radica en las restricciones del material. Las únicas deformaciones que se pueden lograr dentro de los límites de tensión son las que minimizan el estiramiento de las fibras del material. Intentar doblar una hoja de material en dos direcciones resultará imposible (sistema 2D) sin que se produzcan deformaciones plásticas irreversibles, que no son deseables. Este estricto requisito geométrico/material limita severamente el rango de potenciales estructuras arquitectónicas con sistemas de flexión activa a base de placas se reducen a las superficies canónicas desarrollables: cilindros y conos. Para superar tales limitaciones y ampliar la gama de formas realizables, en los casos aquí analizados, ha sido necesario desarrollar soluciones para la inducción de la curvatura Gaussiana. La flexión multidireccional es inducida extrayendo o recortando estratégicamente material y liberando de la restricción de rigidez del entorno a las superficies de material comprometidas.

\subsection{Berkeley Weave}

El primer caso de estudio investiga el potencial de diseño que surge de la integración de la flexión y de la torsión en tiras delgadas de contrachapado. La forma particular es una geometría anticlastica, con una curvatura localmente alta. El proceso de conversión de la forma digital en una estructura de placas plegadas siguió varias operaciones. La primera fue aproximar y discretizar la superficie con una malla cuadrangular (PQmesh). (Fig. 2.b). El análisis de la curvatura de la malla resultante revela que los quads individuales no son planos sino espacialmente curvos (Fig. 2.c). La planaridad de los quads, sin embargo, es una precondición importante para el proceso de fabricación y montaje posterior. En una segunda etapa, la malla se transformó en un patrón de tejido de cuatro capas con tiras y agujeros. Aquí, cada quad se convirtió en un cruce de dos tiras en una dirección que se intersecan con otras dos tiras en un ángulo de 90 grados. Los componentes de la malla entretejida se desarrollaron (unfold) para transformarlos en superficies planares mecanizables. Sin embargo, sólo las regiones donde las tiras están solapadas resultaron planas (áreas azules), mientras que los quads entre las intersecciones permanecieron curvos. (Fig. 2.d). 


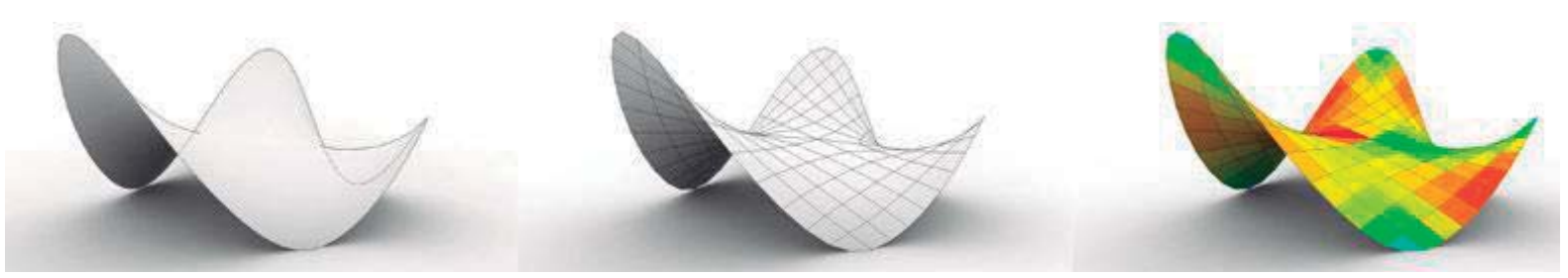

Figura 2 (a-f): Proceso Integral de generación y análisis Digital.

Figura 5.a: Geometría de base. Figura 5.b: aproximación a malla cuadrangular (PQmesh).

Figura 5.c: análisis de la curvatura de la malla.

Un segundo análisis de curvatura ilustra el procedimiento y muestra curvatura cero solamente en las intersecciones de las tiras mientras que los brazos de conexión están curvados y torcidos (fig. 2.e). En el último paso, este modelo geométrico optimizado se utilizó para generar un modelo de fabricación que incluye todos los detalles de conexión y subdivisiones de tiras (fig. 2.f).
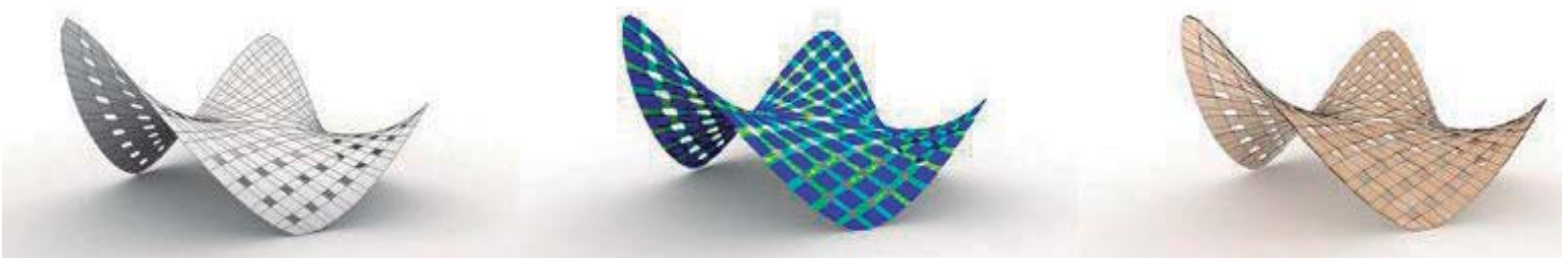

Figura 2.d: Conversión a placas sometidas a flexión activa. Figura 2.e: Análisis de Elementos

Finitos. Figura 2.f: Modelo para Fabricación
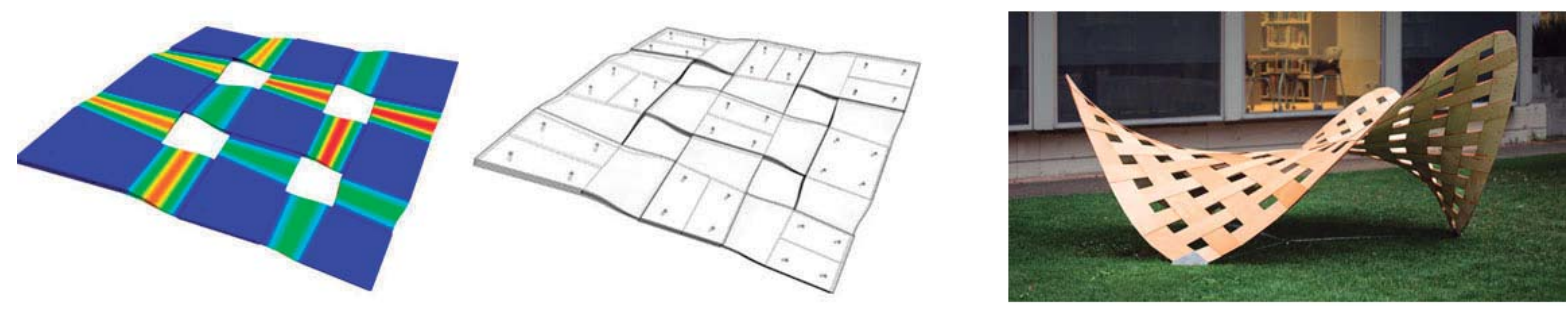

Figure 3.a: Análisis de la curvatura Gaussiana. Figure 3.b: Esquema del tejido y detalles técnicos. Figura 3.c: Vista de la instalación plywood Berkeley Weave construida.

Fuente: Figuras 2 y 3: http://designinnovation.berkeley.edu/wordpress/?portfolio=berkeley-weave

Una mirada más cercana a las regiones más extremadamente curvadas de la estructura ilustra la complejidad relacionada con este último paso (Fig. 3.a). Para permitir una conexión adecuada, los pernos se colocaron solamente en las regiones planas entre las tiras que se intersecan. Puesto que las tiras están compuestas por segmentos pequeños, también fue importante controlar su posición local dentro del tejido. Se creó un patrón que garantizaba que los segmentos de tira sólo terminaban en capas dos y tres y eran sujetados por tiras continuas en capas uno y cuatro. Un efecto secundario positivo de esta estrategia de tejido es que las separaciones entre segmentos nunca son visibles y las tiras parecen estar hechas de una sola pieza. El inconveniente, sin embargo, es que cada segmento tiene una longitud única y requiere posiciones específicas de los orificios de tornillo (fig. 3.b).

Para demostrar el concepto de este enfoque de diseño, este caso de estudio fue construido en dimensiones de $4 \mathrm{~m} \times 3,5 \mathrm{~m} \times 1,8 \mathrm{~m}$ (Fig. 3.c). La estructura se monta con 480 tiras de madera contrachapada geométricamente diferentes sujetas junto a 400 remaches metálicos. El material utilizado es madera contrachapada de abedul de $3,0 \mathrm{~mm}$ de grosor. Se utilizaron las dimensiones y especificaciones de material para un análisis de elementos finitos utilizando el software SOFiSTiK. 


\subsection{Bend9}

El segundo estudio de caso es un arco de varias capas que se extiende más de 5,20 m y tiene una altura de $3,50 \mathrm{~m}$. Este proyecto fue construido para probar la viabilidad técnica de usar placas de flexión activa para estructuras de carga mayores. En comparación con el estudio del caso anterior, este proyecto muestra un patrón de mosaico diferente y explora la posibilidad de aumentar significativamente la rigidez de una forma mediante la interconexión de capas distantes entre sí.

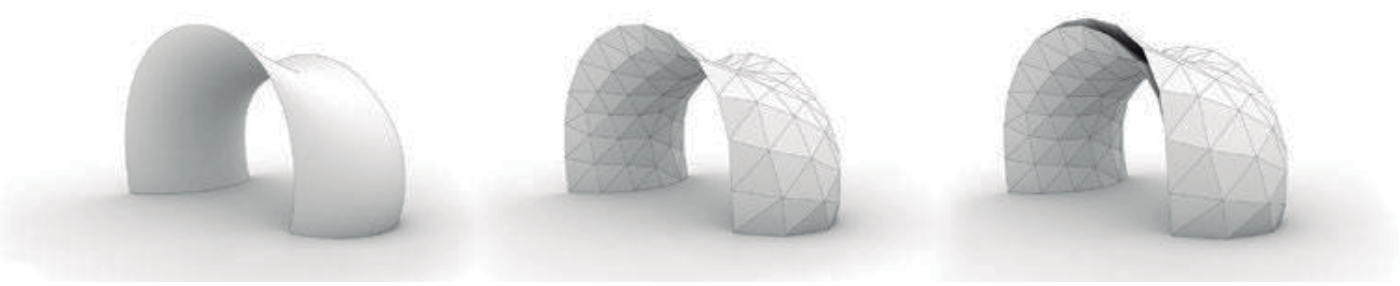

Figura 4 (a-f): Proceso Integral de generación y análisis Digital.

Figura 4.a: Geometría de base. Figura 4.b: aproximación a Mesh. Figura 4.c: Desplazamiento de doble capa (layer offset).

Para aprovechar al máximo las grandes deformaciones que permite el contrachapado, el grosor de las láminas tuvo que reducirse al mínimo, lo que llevó una vez más a la elección radical de emplear madera contrachapada de abedul de 3,0 mm. Dado que las láminas resultantes son muy flexibles, es necesario obtener una rigidez adicional al dotar a la envoltura global de una geometría peculiar que funcione sin problemas como un superficie de curvatura positiva (similar a una esfera) y en otro sentido a una superficie de curvatura negativa (parecida a una silla de montar) (fig. 4.a). Esta pronunciada doble curvatura proporcionó rigidez adicional y evitó modos de deformación indeseables de la estructura. A pesar de la considerable rigidez obtenida a través de la forma, la elección de usar láminas extremadamente delgadas de madera contrachapada requirió refuerzo adicional para proporcionar una resistencia de carga adicional. Estas necesidades fueron satisfechas mediante el diseño de una estructura de doble capa con dos conchas cruzadas.

Como en el ejemplo anterior, la primera etapa del proceso fue convertir la geometría de la base en un patrón de malla (fig. 4.b). En el siguiente paso se realizó un análisis preliminar de la estructura, y se creó una segunda capa mediante la compensación de la malla. Como la distancia entre las dos capas varía para reflejar el momento de flexión calculado a partir del análisis preliminar, el desplazamiento de las superficies cambia a lo largo del tramo del arco (fig. 4.c). El desplazamiento refleja el estado de tensión en las capas individuales, y la distancia entre ellas crece en las áreas críticas para aumentar la rigidez global del sistema. La siguiente lógica de subdivisión de malla que se utilizó para ambas capas garantiza que cada componente pueda doblarse en la forma específica requerida para construir toda la superficie. Esto se logra colocando estratégicamente los huecos en posiciones de destino de la geometría maestra, como se describe en la sección 3, y asegurando así que el proceso de curvado puede tener lugar sin perjuicio de los componentes individuales (fig. 4.d).
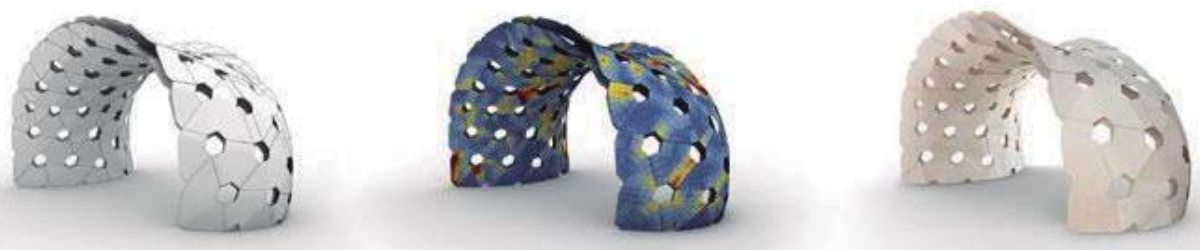

Figure 4.d: Conversión a placas sometidas a flexión activa. Figure 4.e: Análisis de Elementos Finitos. Figure 4.f: Modelo para Fabricación.

Fuente: http://designinnovation.berkeley.edu/wordpress/?portfolio=berkeley-weave 
Aunque inicialmente plana, cada elemento experimenta flexión multidireccional y se bloquea en posición una vez que los componentes adyacentes se agregan al sistema. Los elementos de contrachapado de 3,0 mm proporcionan una rigidez consistente una vez ensamblados, conservando una rigidez sustancial de carcasa. Esto fue validado en un segundo análisis de elementos finitos que consideró tanto el peso propio como los escenarios de carga indeseables (Fig. 4.e). Finalmente, se generó un modelo de fabricación y se fabricó la estructura (Fig. 4.f).

La estructura construida emplea 196 elementos únicos en forma y geometría. Se utilizaron 76 perfiles de madera de sección cuadrada de $4 \mathrm{~cm} \times 4 \mathrm{~cm}$ para conectar las dos capas de madera contrachapada, cuya longitud varia en relación al esfuerzo local. Toda la estructura pesa sólo 160 $\mathrm{kg}$, una característica que también destaca la eficiencia del sistema y su potencial para construcciones ligeras. La transición de curvatura suave y la complejidad global de la forma enfatizan claramente el potencial de la lógica de construcción que se aplica a cualquier tipo de superficie de forma libre de doble curvatura.

\subsection{SSFS, (Same Slope - Folded Surface). Santa Fe 2015}

Construido en la Facultad de Arquitectura, Diseño y Urbanismo de la Universidad Nacional del Litoral (FADU-UNL) en el marco de la Maestría en Arquitectura, Módulo: Proyectación y Construcción Digital, bajo la dirección del Dr. Arq. Mauro Chiarella y el Dr. Arq. Andrés Martín-Pastor, docente e investigador de la Universidad de Sevilla. La experiencia del Pabellón efímero de madera se materializa a partir del trabajo conjunto entre dos universidades (USevilla-UNL).

El fundamento geométrico se encuentra en la teorización y uso de las superficies desarrollables como metodología para la generación de las formas complejas y su transferencia a la arquitectura, en un intento por explorar otras variantes formales no canónicas. Estas lógicas formales requieren para la resolución de su complejidad, tanto un pensamiento Gráfico Aumentado, que evidencia lo insuficiente de la Geometría Descriptiva Tradicional, de Monge, como el instrumental adecuado: el diseño paramétrico que introduce la geometría desde una visión matemático-algorítmica, donde la geometría analítica encuentra el nivel de desarrollo gráfico suficiente. El desarrollo bidimensional de superficies tridimensionales, se logra a partir del principio de la flexión activa en envolventes laminares y posibilita repensar con creatividad métodos e instrumentos de ideación y fabricación en arquitectura. La lógica del SSFS parte de concebir superficies de igual pendiente que se apoyan en diferentes arcos de elipses contenidos en el plano horizontal.

Este caso de estudio es de especial interés ya que se ha participado de manera directa en su concreción material. En el pabellón SSFS la delgada lamina multilaminada de madera guatambú (de 3 capas) de $6 \mathrm{~mm}$ de espesor cubre una distancia de aprox. $9 \mathrm{mts}$ mediante una deformación física aplicada por pliegue material, sometido a flexión activa. Las variables geométricas, parametrizadas computacionalmente, se estudiaron gráficamente. El resultado es una única envolvente continua auto-portante de gran resistencia estructural que ha resistido ráfagas de viento de más de $50 \mathrm{~km} / \mathrm{h}$ (sudestadas y pamperos de la región Litoral Centro argentino). El SSFS expresa lo complejo en su forma resultante y no la construcción y/o montaje de la misma. El enfoque de diseño combina un enfoque basado en el comportamiento con el basado en la geometría. Se parte del conocimiento de los fundamentos de la geometría una y se avanza hacia una aproximación empírica en cuanto al sometimiento del material a los esfuerzos de la pretensión. La falta de especificación de las propiedades intrínsecas del material, como condición de partida, ha determinado que el enfoque integral, entendido como aproximación deseable para el diseño orientado a la optimización, resulte inviable sin las posibilidades de informar al modelo paramétrico con los datos precisos del material.

El trabajo de los alumnos en el Módulo de la Maestría consistió, entre otras tareas, en el montaje mismo del pabellón y en función del diagnóstico: limitaciones contextuales observadas en las 
plataformas utilizadas, se propuso el estudio de una solución alternativa de apoyo que permita flexibilidad y transportabilidad en el montaje de pabellones efímeros. La propuesta fue incorporar tensores de acero, como solución técnico-constructiva eficiente, económica, de montaje fácil y limpio. Los tensores garantizan la rigidez del módulo sin la necesidad de sujeciones y anclajes que dañaran la superficie de apoyo y además, en su dimensión exacta, evitan el trabajo engorroso del replanteo y minimizan al máximo los errores de medición. Optimizan recursos materiales en su dimensionamiento preciso y no altera la lógica constructiva del pabellón. En septiembre de 2015, la propuesta presentada es implementada en la estructura construida en Sevilla por el FabLab-Sevilla, en el contexto de la Noche Europea de los Investigadores.

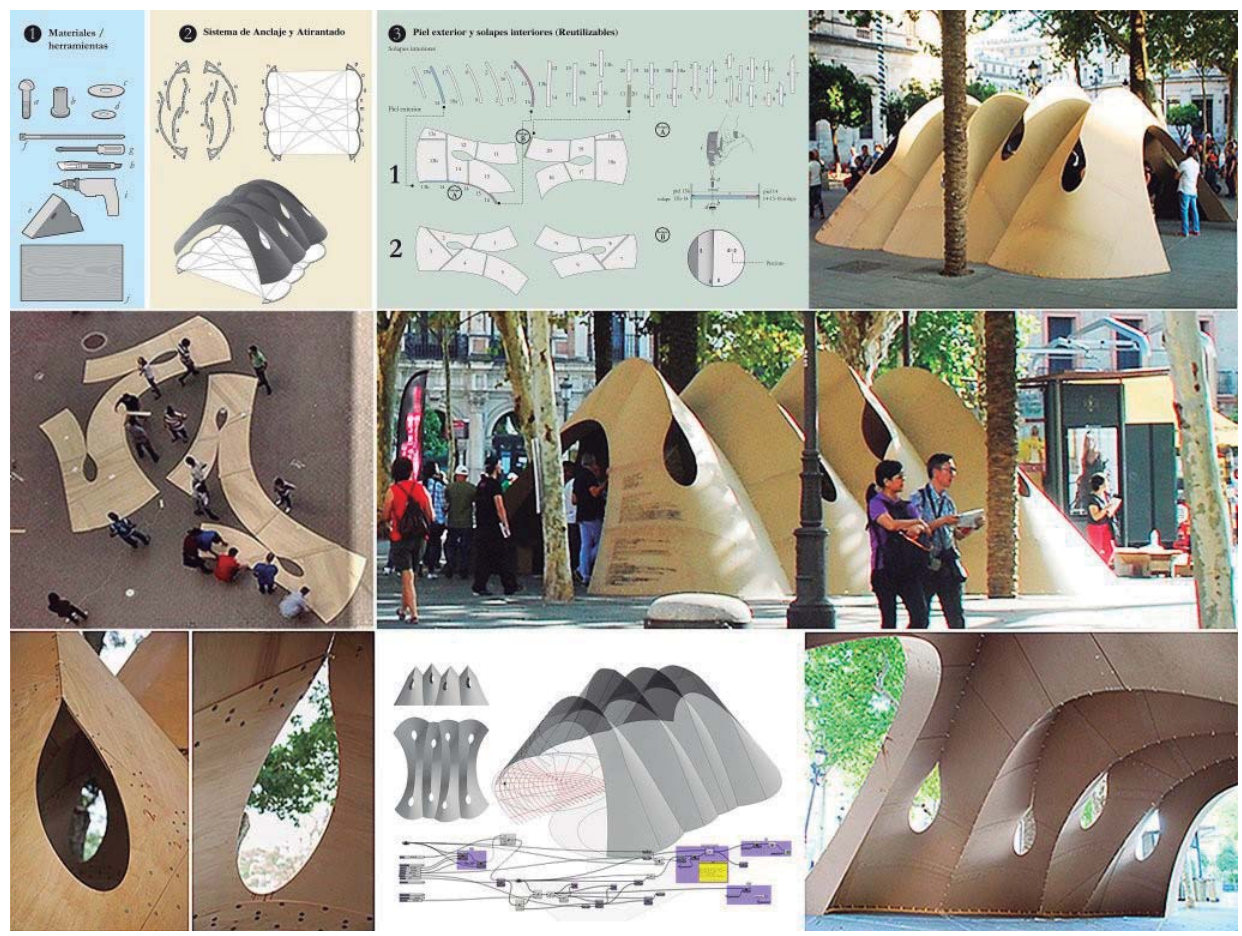

Figura 5: SSFS Pavilion. FADUUNL. Santa Fe. 2015.

Fuente: Chiarella, Mauro; Martín-Pastor, Andrés. SiGraDi, 2015

\section{Conclusiones}

Analizar, identificar y experimentar las ventajas del Diseño orientado a la Optimización, mediante un enfoque Integral y la instrumentalización de la flexión activa en estructuras laminares permite, en primer lugar, construir una mirada crítica sobre el avance de los procesos computacionales en la arquitectura. Por otro lado, en su análisis cuantitativo, contribuye a definir los verdaderos aportes de este enfoque en la optimización de los recursos. El reto clave continúa siendo cómo evaluar tanto la forma global como las características locales de las partes constitutivas de las estructuras, en las que la geometría y las propiedades intrínsecas del material inevitablemente afectan de manera conjunta.

En la propia experiencia se ha tenido que trabajar sin contar con información técnica rigurosa del material utilizado, las investigaciones y desarrollos futuros que pretendan abordar un enfoque integral deberán prever el trabajo conjunto e interdisciplinario con ingenieros en materiales.

\section{Referencias}

ARAYA, Sergio, A. Performative architecture. Thesis (Ph.D.). Massachusetts Institute of Technology, Dept. of Architecture, 2011 . Cataloged from PDF version of thesis. Recuperado de http://hdl. handle.net/1721.1/68413.

CHIARELLA, Mauro; MARTíN-PASTOR, Andrés. "Pensamiento Gráfico y Diseño Colaborativo. Geometrías desarrollables en composiciones plegadas". XIX Congreso de la Sociedad de Gráfica Digital (SiGraDi). Florianópolis, Santa Catarina, Brasil. 23 a 27 de Noviembre 2015.

HENSEL, Michael; MENGES, Achim; WEINSTOCK, Michael. (eds). "Frei Otto in Conversation with the Emergence and Design Group". En: Emergence: Morphogenetic Design Strategies, AD. Architectural Design. Wiley. UK, 2004. p. 19-25.

HENSEL, Michael Ulrich; MENGES, Achim; WEISNTOCK, Michael; (Eds.). Techniques and Techno- 
logies in Morphogenetic Design. AD. Architectural Design. Wiley. UK, 2006.

HENSEL, Michael Ulrich; MENGES, Achim, (Eds.). Versatility and Vicissitude. Performance in Morpho-Ecological Design. AD. Wiley, 2008.

HENSEL, Michael Ulrich; AD Primer: Performance-oriented Architecture - Rethinking Architectural Design and the Built Environment. Londres: AD Wiley. Kindle Edition, 2013.

KOHLER, Matthias; GRAMAZIO, Fabio. Digital Materiality in Architecture, Lars Müller Publishers, 2008.

KOLAREVIC, Branko, (ed.), Architecture in the Digital Age Design and Manufacturing, Spon Press, London, UK, 2003.

KOLAREVIC, Branko. 'Computing the Performative in Architecture', Proceeding of the eCAADe

21 conference. Graz, Austria, 2003. p. 195.

KOLAREVIC, Branko; MALKAWI, Ali, (Eds.), Performative Architecture: Beyond Instrumentality. London, UK: Spon Press, 2005.

LIENHARD (et al). "Active Bending, A Review on Structures where Bending is used as a Self-Formation Process". Artículo en: International Journal of Space Structures. Diciembre, 2013. DOI: 10.1260/0266-3511.28.3-4.187. Disponible en: https://www.researchgate.net/publication/261596411

LIENHARD, Julian. Bending Active Structures, Form-Finding Strategies Using Elastic Deformation In Static And Kinematic Systems And Structural Potential Therein, Phd Thesis, ITKE, University Of Stuttgart, 2014.

LIENHARD, Julian; SCHLEICHER, Simon; KNIPPERS, Jan. "Bending-Active Structures - Research Pavilion ICD/ITKE. In Proceedings of the International Symposium of the IABSE-IASS Symposium, Taller Longer Lighter, Nethercot, D., Pellegrino, S. et al. (eds). London: Brintex Digital Publishing. 2012.

OTTO Frei; RASCH, Bodo. Finding Form: Towards an Architecture of the Minimal, Ediciones Axel Menges, Germany, 1 ed. 1995. 240 p. Disponible en: https://es.scribd.com/doc/128383232/ Frei-Otto-Bodo-Rasch-Finding-Form

OXMAN, Neri. Material Based Design Computation, PhD Dissertation: Massachusetts Institute of Technology, Junio, 2010.

OXMAN, Rivka. "Performance-based Design: Current Practices and Research Issues". International journal of architectural computing, Londres. v. 08 n.3, 2008. P. 337 - 358. Doi: $10.1260 / 147807708784640090$. Disponible en: https://cumincad.architexturez.net/doc/ oai-cumincadworks-id-ijac20076101

PICON, Antoine. "Arquitectura y virtualidad. Hacia una nueva condición material". En: Revista Praxis 6. New technologies: New architectures. [en linea] Pontificia Universidad Católica de Chile. Santiago, Chile: ARQ, 2006. [19 de junio de 2017] Disponible en: $<$ http://www.redalyc.org/articulo.oa?id=37506302> ISSN 0716-0852

SCHLEICHER, Simon; RASTETTER, Andrew; LA MAGNA, Riccardo; SCHÖNBRUNNER, Andreas; HABERBOSCH, Nicola and KNIPPERS, Jan. "Form-Finding and Design Potentials of Bending-Active Plate Structures." In Modelling Behaviour, 2015. pp. 53-63. Switzerland: Springer. December 2015.

SENNETT, Richard. El artesano. Barcelona, España: Anagrama, 2009. 416 p. 